Conclusions: CCL18 elevates especially in synovial fluid of RA patients, which may correlate with one-year radiographic progression through promoting migration of RA-FLS.

Acknowledgements: This work was supported by National Natural Science Foundation of China (grant no. 81671612, 81601427) and Natural Science Foundation of Guangdong Province (grant no. 2016A030313307, 2017A030313470).

Disclosure of Interest: None declared

DOI: 10.1136/annrheumdis-2018-eular.6704

\section{THU0118 PATIENT-AND PHYSICIAN-REPORTED BARRIERS TO ACHIEVING RHEUMATOID ARTHRITIS (RA) DISEASE CONTROL}

M.I. Danila ${ }^{1}$, L. Chen ${ }^{1}$, R. O'Beirne ${ }^{2}$, E.M. Ruderman ${ }^{3}$, L.R. Harrold ${ }^{4}$, J.A. Melnick ${ }^{1}$ M.M. Safford ${ }^{5}$, J.M. Kremer ${ }^{6}$, J.R. Curtis ${ }^{1} .{ }^{1}$ Medicine: Rheumatology, ${ }^{2}$ Continuing Medical Education, University of Alabama at Birmingham, Birmingham; ${ }^{3}$ Medicine: Rheumatology, Northwestern University, Chicago; ${ }^{4}$ Medicine: Orthopedics and Physical Rehabilitation, University of Massachusetts Medical School, Worchester, ${ }^{5}$ Medicine, Cornell University, New York City; ${ }^{6}$ Corrona LLC, Waltham, USA

Background: Many patients with RA do not achieve guideline-recommended treat-to-target (T2T) goals in clinical practice. Little is known about the challenges that patients and rheumatologists face when attempting to achieve better control of RA disease activity.

Objectives: To identify and prioritise patient- and rheumatologist-perceived barriers to achieving RA disease activity control.

Methods: Participants were recruited from the Consortium of Rheumatology Researchers of North America (Corrona) registry and invited to participate in nominal groups (NGs), 4 with patients and 3 with rheumatologists. Each group generated a list of barriers to reaching RA disease control (patients) and T2T goals (rheumatologists). Two separate lists containing the generated items were created and were subjected to a card sort procedure to create common themes. A random sample of Corrona RA patients were invited by email to complete a compensated online survey and asked to rank their top 3 barriers. A weighted score was assigned for each barrier by considering the number of respondents who ranked it and the priority rank they assigned. The barriers/themes were sorted into domains. The patient survey also included knowledge items about T2T strategy and attitudes about RA treatment.

Results: Nominal groups with 37 RA patients identified 17 themes to achieving control of RA activity. Similarly, 8 themes emerged from the physician NGs, 7 of which were also found in the patient NGs (table 1). Cost of RA care was ranked highest by both patients and physicians, while medication risk aversion ranked second and third among the physician- and patient-generated barriers, respectively. We sent 1694 invitations to complete the survey and 450 patients with RA for whom clinical data was available responded within 3 weeks. There were no differences in age, sex, or disease duration between survey- respondents and nonrespondents. A higher proportion of respondents were college-educated. A total of $344(77 \%)$ respondents considered RA to be a high priority for their health, 225 (51\%) reported being familiar with T2T as a treatment strategy, and 312 (85\%) displayed core beliefs favouring medications. Among the challenges to controlling RA disease activity, the domain that received the highest score was unpredictability of RA and its treatment (figure 1). Symptoms and illness burden domain received the second highest score, followed by the health system factors domain (figure 1).

Abstract THU0118 - Table 1. Common Patient- and Physician- Reported Barriers to Achieving RA Control Identified by Nominal Groups (NGs)

\begin{tabular}{lcc}
\hline Barriers (Themes) & \multicolumn{2}{c}{ Proportion of Total Votes } \\
\cline { 2 - 3 } & Patient NGs & Physician NGs \\
\hline Cost/Administrative & $13.0 \%$ & $27.0 \%$ \\
Medication Effectiveness & $7.4 \%$ & $3.0 \%$ \\
Medical Risk Aversion & $6.5 \%$ & $20.0 \%$ \\
Access to Care & $4.6 \%$ & $8.0 \%$ \\
Medication Adherence & $1.9 \%$ & $9.0 \%$ \\
Patient-Physician Communication & $1.9 \%$ & $12.0 \%$ \\
Education & $0.9 \%$ & $5 \%$ (Physician) \\
\hline
\end{tabular}

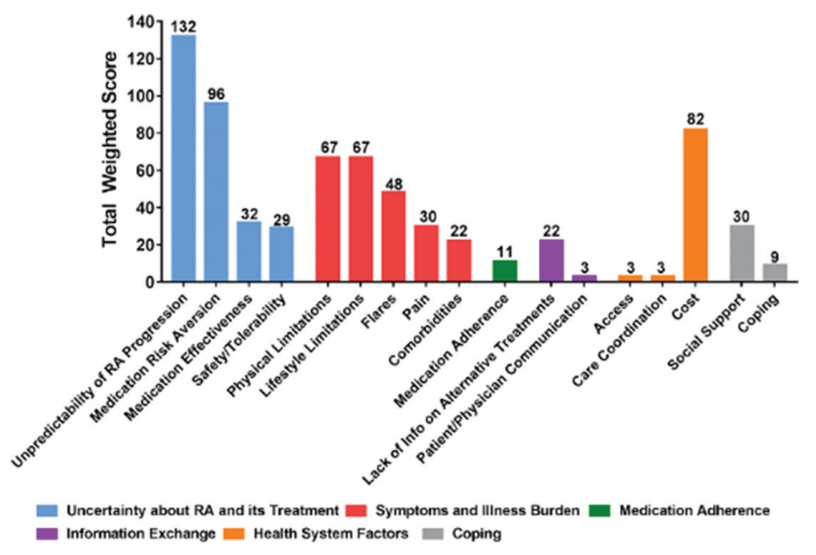

Figure 1. Patient-prioritised Barriers to Acheiving RA Control in Corrona Registry

Conclusions: There are common patient- and physician-perceived barriers to achieving RA disease control. From the patient perspective important barriers are unpredictability of how RA may progress, medication risk aversion and cost of RA care. Addressing these barriers, when possible, may improve goal-directed RA care.

Acknowledgements: Pfizer IGLC, Corrona

Disclosure of Interest: None declared

DOI: 10.1136/annrheumdis-2018-eular.2005

\section{THU0119 SMOKING IS NEGATIVELY ASSOCIATED WITH CLINICAL RESPONSE TO CONCOMITANT PREDNISONE USE IN EARLY RHEUMATOID ARTHRITIS}

M. Safy, M. De Hair, P. Welsing, J. Van Laar, J. Jacobs. Rheumatology and Clinical Immunology, University Medical Center Utrecht, Utrecht, Netherlands

Background: Smoking and overweight increase the risk of development of rheumatoid arthritis (RA) and have been associated with a reduced clinical response to several biological disease modifying anti-rheumatic drugs (bDMARDs) and methotrexate (MTX). ${ }^{1-6}$ The effect of smoking and overweight on clinical response to prednisone (pred) has not been investigated

Objectives: To determine whether smoking and overweight are negatively associated with clinical response to pred in RA, as assessed by the disease activity score using 28 joints (DAS28). Second, to similarly determine the effect of smoking and overweight on the four individual components of DAS28.

Methods: In the second Computer Assisted Management in Early Rheumatoid Arthritis trial (CAMERA-II), patients with early, DMARD naïve RA were randomised to an MTX based treatment strategy with addition of $10 \mathrm{mg}$ pred or placebo daily for 2 years. We used data from the patients concomitantly treated with pred, $n=127$. We performed linear mixed model analyses with DAS28 over the 2 year trial period as outcome variable and smoking status (current yes $/ \mathrm{no}$ ) and BMI (in $\mathrm{mg} / \mathrm{kg}^{2}$ ) as predictor variables, corrected for baseline DAS28, gender, rheumatoid factor (RF-test negative or positive), MTX administration route (subcutaneous or oral), MTX dosage (mg/week) and bDMARD use (yes/no), time (in months) and time ${ }^{2}$. For the second aim, we performed an extended mixed model analysis with a multivariate outcome, consisting of the individual components of the DAS28 (tender joint count 28 , swollen joint count 28 , erythrocyte sedimentation rate and VAS general health) and as predictor variables smoking status and BMI, correcting for important confounders.

Results: Smoking data was available of $104 / 127$ patients. Smoking was significantly associated with higher DAS28 over time, corrected for baseline DAS28, compared to non-smoking (table 1). No significant associations were found between $\mathrm{BMI}$ and response to pred, nor between smoking or BMI and any of the individual components of DAS28.

Table 1 The association between smoking and DAS28 in patients treated with concomitant prednisone for 2 years 